\title{
Tratamiento de la disfunción del suelo pélvico
}

\author{
Pena Outeiriño JM, Rodríguez Pérez AJ, Villodres Duarte A, Mármol Navarro S, \\ Lozano Blasco JM.
}

Servicio de Urología. Unidad de Neurourología y Uroginecología. Hospital Universitario Virgen del Rocío. Sevilla

Actas Urol Esp.2007;31(7):719-731

\section{RESUMEN}

TRATAMIENTO DE LA DISFUNCIÓN DEL SUELO PÉLVICO

El periné está formado por elementos músculos-aponeuróticos que se integran bajo el control del sistema nervioso. Su alteración es responsable de patologías uroginecológicas, coloproctológicas y sexuales. Para conseguir su tratamiento exitoso es obligado no olvidar el papel que juega el periné en las mismas.

El tratamiento de la disfunción del suelo pélvico agrupa una serie de técnicas y procedimientos conservadores como cambios en hábitos de vida, terapia conductual, biofeedback, electroestimulación (neuromodulación y estimulación eléctrica periférica) y entrenamiento con ejercicios musculares del suelo pélvico (rehabilitación perineal).

El objetivo de todas ellas es mejorar o conseguir la continencia urinaria, el fortalecimiento de su musculatura para conseguir equilibrar la estática pélvica, mejorar la vascularización local y la función ano-rectal además de conseguir una sexualidad satisfactoria.

Palabras clave: Disfunción de suelo pélvico. Continencia urinaria. Rehabilitación perineal.

\section{ABSTRACT \\ TREATMENT OF THE DYSFUNCTION OF THE PELVIC FLOOR}

The perineum is formed by muscle-aponeurotic elements that are integrated under the control of the nervous system. Their alterations are responsible for urogynecological, coloproctologic and sexual pathologies. In order to obtain a successful treatment, it is obliged not to forget the role that plays the perineum in those pathologies.

The treatment of the dysfunction of the pelvic floor groups conservative techniques and procedures like changes in life habits, behavioural therapy, biofeedback, electroestimulation (neuromodulation and peripheral electrical stimulation) and training with muscular exercises of the pelvic floor (perineal rehabilitation).

The objective of all of them is to improve or to obtain the urinary continence, the strengthening of its musculature to be able to balance pelvic static, to improve the local vascularization and the anorrectal function besides securing a satisfactory sexuality.

Keywords: Dysfunction of the pelvic floor. Urinary continence. Perineal rehabilitation.

$\mathrm{E}^{1}$ suelo pélvico o periné es un conjunto de músculos que sustentan la porción abdominal inferior y sirven de apoyo a la vejiga, el útero y una porción del intestino. Es fundamental que esta musculatura mantenga una buena función, ya que la debilidad del suelo pélvico es causa de incontinencia urinaria de esfuerzo, cisto y rectocele, prolapsos genitales, disfunciones sexuales (dispareunia, disminución de la sensibilidad e intensidad del orgasmo).

La lesión o debilidad de la musculatura perineal puede estar ocasionada por diversas causas: 
- En el Embarazo, tanto el peso del útero como el efecto relajador de las hormonas pueden debilitar el suelo pélvico.

- El Parto provoca lesiones músculo-aponeuróticas y neurológicas perineales durante el periodo expulsivo. Además los esfuerzos realizados actúan directamente sobre la musculatura del suelo pélvico.

- Falta de estrógenos en posmenopáusicas: provocan pérdida de tono y flacidez de los músculos perineales.

- Intervenciones quirúrgicas sobre el periné.

- Obesidad.

- Estreñimiento.

- Tos crónica del tabaquismo.

- Profesiones de riesgo (deportistas, cantantes, músicos de instrumentos de viento,..).

- Desconocimiento o falta de conciencia de la zona perineal y de su participación en la fisiología sexual, urológica y digestiva.

El conocimiento del papel que juega el suelo pélvico en los mecanismos de la continencia, como elemento de sostén de la unión uretrocervical, su implicación en el sistema esfinteriano estriado, así como las alteraciones neurológicas que conlleva las disfunciones severas del mismo, ha conseguido que se retome con gran interés aquellas técnicas que llevan a restablecer el estado de normofuncionalidad de estas estructuras como forma de tratamiento de las patologias secundarias a dichas alteraciones: incontinencia urinaria de esfuerzo, mixta, de urgencia, disfunciones sexuales, patologías ginecológicas y obstétricas y colo-proctológicas ${ }^{1}$.

Las técnicas de rehabilitación del suelo pélvico como tratamiento de la incontinencia urinaria femenina de esfuerzo (IUE) se vienen imponiendo en estos últimos años después de que el ginecólogo Arnol Kegel demostrará hacia 1950 la estrecha relación que existía entre los escapes de orina y la hipotonía o debilidad de la musculatura perineal, desapareciendo o mejorando notablemente los síntomas cuando se practicaban ejercicios de refuerzo de estas estructuras ${ }^{2}$.

Este tipo de tratamientos no alcanzó relevancia en su momento quizás porque no se terminaban de conocer bien los mecanismos fisiopatológicos de la IUE, no se tenía al alcance la tecnología que disponemos hoy en día y por otro lado quizás no estuvieran todavía bien definidas las distintas pautas y sistemáticas de tratamiento ${ }^{3,4}$.

La continencia urinaria se consigue por una compleja interrelación entre aparato urinario inferior y musculatura perineal bajo el control de mecanismos neurológicos voluntarios y autonómicos.

La musculatura estriada del periné recibe inervación pudenda de carácter voluntario y por tanto susceptible de aprendizaje directo. Contrariamente, la participación del sistema nervioso autonómico sobre el aparato urinario inferior está controlada por la actividad facilitadora o inhibitoria de centros superiores sobre los centros miccionales lumbo-sacros. Este control escapa al aprendizaje directo, aunque puede desarrollarse una vez que los mecanismos voluntarios se establezcan a nivel autonómico. En esencia, aprender es crear vías de facilitación para algún tipo de actividad neurológica. Estas vías se consiguen realizando una y otra vez dicha actividad.

Estos fundamentos constituyen la base de la mayoría de procedimientos para el tratamiento conservador de las disfunciones miccionales secundarias a patología del suelo pélvico.

Las técnicas de rehabilitación perineal y otras pautas para el tratamiento de la disfunción perineal constituyen un apartado muy amplio y específico a desarrollar en las unidades del suelo pélvico, en las que un fisioterapeuta especializado en el tema y conocedor profundo de toda la patología derivada de los defectos funcionales del suelo pélvico debe trabajar en estrecha relación con la unidad urológica que lleve el tema; además sería aconsejable que estas unidades de rehabilitación del suelo pélvico estuvieran integradas dentro del propio servicio de urología con el fin de ajustar al máximo las pautas de tratamiento rehabilitador según el tipo disfunción existente, instaurando tratamiento farmacológico si precisase el caso y derivando a cirugía con criterio y sin demora en los casos necesarios. Nosotros tenemos experiencia en ello y apostamos por esta fórmula ${ }^{5}$.

El tratamiento conservador de la disfunción del suelo pélvico, especialmente de la incontinencia generada por debilidad muscular perineal, agrupa distintas medidas tales como modificación de los hábitos higiénico-dietéticos y terapia conductual, utilización de dispositivos absorbentes, fisioterapia y entrenamiento con ejercicios 
musculares del suelo pélvico. Estos métodos son en general de fácil aplicación, escaso riesgo de efectos secundarios y buena relación coste-beneficio, aunque la evaluación de la eficacia real de estos tratamientos es problemática por el escaso número de estudios controlados publicados.

Los objetivos de este tratamiento conservador son mejorar o conseguir la continencia urinaria, reforzar la musculatura estriada para y periuretral, conseguir un aumento del equilibrio en la estática pélvica, mejorar la vascularización local y la función ano-rectal además de conseguir una sexualidad satisfactoria.

Una vez realizado el balance reeducador y evaluadas las necesidades y posibilidades de cada paciente debe seguirse una pauta recuperadora que constará de la combinación de alguna de las siguientes técnicas: Modificación del estilo de vida y psicoterapia; reeducación vesical y vaciado puntual; rehabilitación del suelo pélvico; conos vaginales (cinesiterapia); biofeedback; electroestimulación y neuromodulación.

\section{MODIFICACIONES EN EL ESTILO DE VIDA Y PSICOTERAPIA}

Consisten en unas serie de medidas higiénicodietéticas, que incluyen: mantener un peso adecuado para reducir la sobrecarga del suelo pélvico, evitar ejercicios intensos con aumentos de la presión intraabdominal, disminuir la ingesta de xantinas (café, té), regularizar el ritmo intestinal, modificar el entorno para facilitar la micción.

Este sea posiblemente el menos conocido y el menos utilizado de los tratamientos conductuales de la disfunción del suelo pélvico, aunque ningún terapeuta duda que la incontinencia lleva asociados importantes trastornos psicológicos del tipo de ansiedad, depresión e incluso histeria. Estas pacientes tienen también con frecuencia disfunciones sexuales. Todos estos trastornos se presentan con mayor frecuencia e intensidad que en las mujeres no incontinentes ${ }^{5}$. La psicoterapia se realiza en sesiones semanales de 50 minutos hasta completar un total de 8-12 sesiones; aproximadamente la mitad inicial son individuales para finalizar el tratamiento con sesiones en grupo; las sesiones se orientan a reducir la ansiedad, a enseñar medidas para soportar mejor los sintomas y a combatir la depresión.

\section{REEDUCACIÓN VESICAL Y VACIADO PUNTUAL}

\section{Reentrenamiento vesical}

El reentrenamiento vesical tiene como objetivo principal incrementar el tiempo entre cada micción voluntaria mediante una agenda individualizada y ajustada para cada enferma, se emplea para la incontinencia por urgencia y la inestabilidad vesical y también para tratar el componente de urgencia de la incontinencia urinaria mixta. Son necesarios varios meses de tratamiento hasta alcanzar los objetivos, también es preciso que las pacientes tengan una esfera cognitiva íntegra y físicamente capaces de desarrollar tareas rutinarias; deben estar motivadas para cumplir el régimen de tratamiento. El reentrenamiento vesical comprende tres componentes, educación a la paciente, agenda de vaciado y refuerzo positivo. La educación de la paciente previa a entrar en el programa es fundamental; y el refuerzo positivo lo constituye el descenso de los episodios de incontinencia, de urgencia y los ánimos del terapeuta $^{6,7}$. La primera visita de la paciente una vez decidido el empleo del reentrenamiento vesical se basa en la enseñanza de la fisiología y patofisiología de la micción, la paciente debe comprender su problema y las posibles soluciones, debe informársele de que los beneficios irán siendo graduales y siempre obtenidos con su propio esfuerzo y de que nosotros no seremos sino meros educadores y ayudantes de ella, que será la persona encargada de curarse por sí misma. En esta primera visita se le enseña a rellenar el diario miccional y se le instruyen en algunas técnicas de relajación. La hora de la micción se irá retrasando semana a semana progresivamente, comenzando por intervalos pequeños, que deben ser ajustados para cada paciente y que pueden ir alargándose de quince en quince minutos. El objetivo final es que la paciente orine cada tres o cuatro horas y que desaparezcan los episodios de incontinencia por urgencia. La duración del programa es variable para cada paciente y oscila alrededor de seis semanas. El diario miccional consiste en una hoja desglosada en horas, con columnas para las micciones, los escapes, los episodios de urgencia, hora de levantarse y acostarse, el número de absorbentes, fecha y comentarios. Los porcentajes de curación difieren mucho 
según las series y según la definición de éxito o fracaso pero oscilan alrededor del $60 \%{ }^{8,9}$.

\section{Vaciado puntual}

El vaciado puntual, a diferencia del anterior, no requiere indemnidad cognitiva de la paciente, por ello no precisa de tanta colaboración como el anterior y se usa especialmente para pacientes incontinentes con demencias $\mathrm{u}$ otros deterioros mentales. El objetivo consiste en mantener vacía la vejiga y seca a la paciente ${ }^{10}$. En este programa son necesarias varias visitas para explicar a la paciente los objetivos, enseñarle a vaciar su vejiga, relajada, con prensa abdominal, con maniobra de Credé, etc. y a lograr ir sola al baño. Una vez logrado esto, se le establece un programa en el que comenzará vaciando su vejiga cada dos horas desde que se levanta hasta que se acuesta durante la primera semana; en la visita de control, si la paciente no ha tenido escapes, se aumentan los tiempos de vaciado; si está mojada, se disminuyen de media en media hora hasta conseguir reducir al mínimo los escapes. Las revisiones sistemáticas acerca del vaciado puntual no encuentran suficientes evidencias para establecer conclusiones acerca de este tratamiento no obstante parece ofrecer beneficios a corto plazo en la incontinencia urinaria en adultos ${ }^{11}$. Los resultados parecen ofrecer aproximadamente un $50 \%$ de respuesta definida ésta como permanecer seca o con un episodio de escape al día ${ }^{12}$.

\section{REHABILITACIÓN DEL SUELO PÉLVICO}

Los mecanismos responsables de la continencia urinaria, tanto en reposo como en situaciones de esfuerzos, son diversos.

Los elementos músculo aponeuróticos del periné aportan un adecuado soporte a la unión vésico-uretral y participan activamente en el sistema esfinteriano periuretral.

La musculatura perineal la componen dos tipos de fibras musculares ${ }^{13}$ : fibras de contracción lenta (tipo I) responsables del tono muscular y soporte de órganos y fibras de contracción rápida (tipo II) localizadas predominantemente a nivel del esfinter estriado periuretral, responsables junto con los elementos aponeuróticos del cierre uretral durante los incrementos de presión abdominal.
La misión de la rehabilitación perineal es tonificar y fortalecer ambos tipos de fibras; las de fibras lentas con contracciones suaves y mantenidas y las de fibras rápida con contracciones de mayor amplitud (fuertes) y rápidas.

El efecto final del tratamiento rehabilitador es:

- Incremento de las unidades motoras.

- Mejora de la frecuencia de excitación.

- Incremento de la masa muscular.

La puesta en marcha de un programa de rehabilitación requiere 3 condiciones imprescindibles, en las que el fisioterapeuta de la unidad juega un papel fundametal para su consecución:

1. Gue la paciente tenga conciencia de su periné.

2. Que logre aislar los grupos musculares que nos interesa fortalecer, relajando la musculatura parásita.

3. Motivación en la mujer para conseguir un buen aprendizaje y un adecuado cumplimiento del trabajo encomendado.

Además, existen una serie de factores que influyen en el éxito de la rehabilitación perineal, a saber:

1. Grado de incontinencia.

2. Edad.

3. Integridad esfinteriana.

4. Tono perineal.

5. No antecedentes quirúrgicos ni radioterápicos.

6. Fuerte motivación de la mujer.

7. Perseverancia en el trabajo.

8. Personal cualificado.

9. Seguimiento a corto y medio plazo.

Las técnicas o procedimientos de nuestro programa de rehabilitación se dividen en activos o pasivos, dependiendo de la participación voluntaria de la paciente en los mismos.

En los activos tenemos la Cinesiterapia verbal, Cinesiterapia con biofeedback y los ejercicios con conos vaginales.

La rehabilitación pasiva se realiza con técnicas de electroestimulación. Entre los activos, la cinesterapia verbal o gimnasia perineal es la técnica más simple ${ }^{14}$. Requiere un tiempo de aprendizaje individual para que la mujer reconozca y logre aislar los grupos musculares a rehabilitar, pasando posteriormente a realizar de forma repetitiva una tabla de ejercicios en su domicilio. 
Para facilitar su aprendizaje podemos adiestrar verbalmente a la paciente a que en el momento de la micción contraiga repetidas veces la musculatura del periné intentando cortar o modificar el chorro de orina, evitando contraer glúteos o rectos, hasta que tome conciencia de los músculos a contraer.

$\mathrm{Al}$ no requerir ningún tipo de aparataje se convierte en la técnica más económica siendo ideal para su aplicación en atención primaria, en programas de profilaxis de incontinencia urinaria, para recuperación perineal tras el parto y para casos de incontinencia urinaria leve.

El problema de esta técnica es que no disponemos de datos objetivos para comprobar su correcta ejecución. Para ello podemos aplicar técnicas de biofeedback.

Con el biofeedback los ejercicios musculares del suelo pélvico se realizan asistidos por un equipo más o menos sofisticado que traduce la contracción muscular en una señal gráfica, acústica o ambas, para que el paciente y el fisioterapeuta perciban el trabajo realizado.

Las ventajas del biofeedback en la rehabilitación perineal son varias ${ }^{15,16}$ :

1. Facilita el aprendizaje porque ayuda al reconocimiento de las estructuras del suelo pélvico, facilitando su aislamiento e integrándolas en los mecanismos conscientes.

2. Posibilita la autoevaluación del trabajo que se realiza.

3. Creando una fuerte motivación para la paciente.

4. Es posible el control por el fisioterapeuta del trabajo realizado.

5. Se puede programar un trabajo personalizado de acuerdo a las necesidades de la paciente.

Así cuando el problema a solucionar es una IUE nuestra prioridad es reforzar la función de sostén, trabajando fundamentalmente sobre las fibras lentas para conseguir un tono perineal adecuado. Además se debe fortalecer las fibras de contracción rápida para asegurar la continencia urinaria durante los esfuerzos.

En casos de incontinencia urinaria de urgencia nuestra misión es reforzar las fibras del esfinter estriado cuya contracción voluntaria, coincidiendo con los episodios de urgencia, activen el reflejo inhibitorio perineo-detrusoriano.
La introducción del biofeedback en las técnicas de rehabilitación perineal ha mejorado significativamente sus resultados, siendo la técnica más eficaz para la recuperación perineal.

Nuestra experiencia durante 4 años hemos tratado con rehabilitación del suelo pélvico 412 mujeres con incontinencia urinaria. La edad media fue de 49,7 años con una desviación de 10,3 y un rango de 14 a 72 años.

Incontinencia urinaria de esfuerzo la presentaban 189 pacientes $(45,9 \%)$ mientras que 223 $(54,1 \%)$ tenían además un componente de urgencia etiquetándose por ello como mixtas. No se incluyeron incontinencias de urgencia puras.

Los resultados obtenidos, en relación a la persistencia o no de la incontinencia, fueron definidos como: éxito, aceptable o fracaso (Fig. 1 ).

Para considerar que una paciente ha tenido éxito con la rehabilitación debe comunicarnos que no presenta escapes a los esfuerzos y que no usa absorbentes.

El resultado aceptable correspondería a pacientes con clara disminución de escapes y del uso de absorbentes.

Cuando los criterios anteriores no se cumplen o la paciente abandona el programa por malos resultados se etiquetan como fracasos.

Globalmente, al mes de seguimiento los éxitos se acercaron al 50\%, los resultados aceptables superaron la tercera parte de nuestras pacientes y el fracaso apareció en el 15,3\% de la muestra.

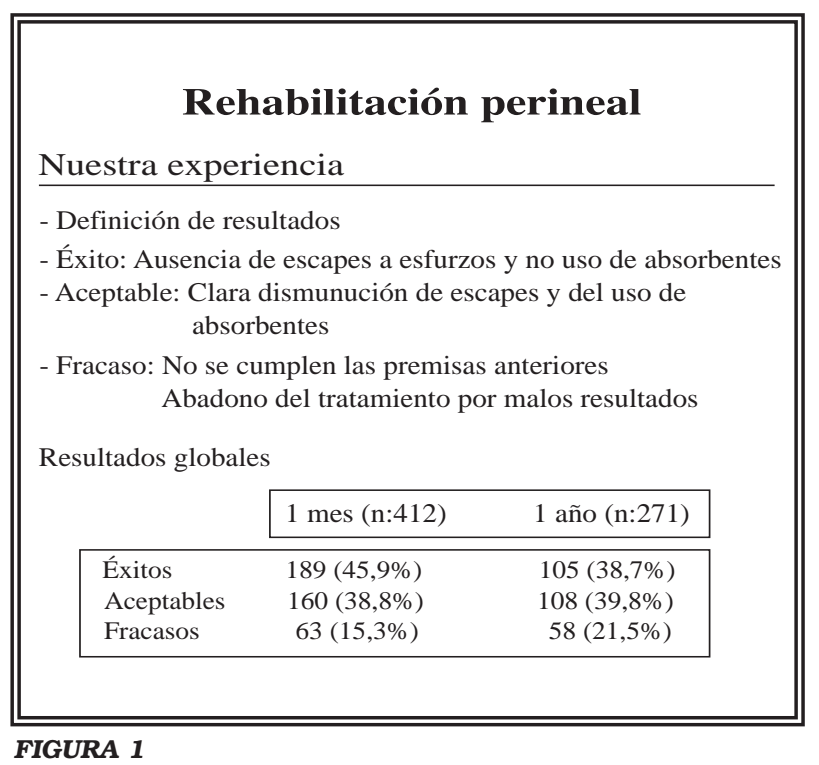


Cumplieron el año de seguimiento 271 pacientes. Los resultados exitosos y aceptables se igualaron en torno al 38-40\% cada uno. Los fracasos se incrementaron al 21,5\%.

$\mathrm{Si}$ analizamos los resultados obtenidos en función al grado o severidad de la incontinencia se observó que las tres cuartas partes de pacientes con incontinencia leve tuvieron éxito con la rehabilitación. En casos de incontinencia moderada los resultados aceptables, con un 51\%, superaron ligeramente a los éxitos. En incontinencias severas los fracasos dominaron con cerca del 70\% de casos, siendo los éxitos menores al $3 \%$.

Cuando en esta misma relación sólo se incluyen pacientes que realizan bien los ejercicios los porcentajes de éxitos se incrementan en todos los grados de incontinencia, sobre todo en las leves y moderadas que pasan del 74 al 95\% y de 42 al $56 \%$ respectivamente.

Si valoramos la calidad de vida y el estado de ánimo de nuestras pacientes en relación a la incontinencia urinaria antes y después de la rehabilitación perineal, observamos una mejoría de la calidad de vida y autoestima en todos los grupos analizados.

Finalmente vemos la repercusión que nuestro programa de rehabilitación tuvo en la programación quirúrgica de la incontinencia urinaria de esfuerzo. Desde la aplicación de la rehabilitación perineal para el tratamiento de la incontinencia urinaria de esfuerzo el número de su cirugía en nuestro hospital se ha reducido un $30-35 \%$, con las consiguientes repercusiones económicas en el presupuesto hospitalario (Fig. 2).

\section{Otra técnica de rehabilitación activa es la gimnasia perineal con conos vaginales}

Utiliza dispositivos lastrados en forma de cono para su aplicación intravaginal, Cada cono tiene un peso diferente gradual (aunque son del mismo tamaño), y se usan manteniéndolos en la vagina como un pequeño tampón durante unos minutos al día, de pie o caminando. Al introducir el cono en la vagina, tiende a descender y caer por su propio peso. La sensación de pérdida del cono provoca un suave reflejo de contracción en los músculos del suelo pélvico que hace retener el cono. Esta sencilla

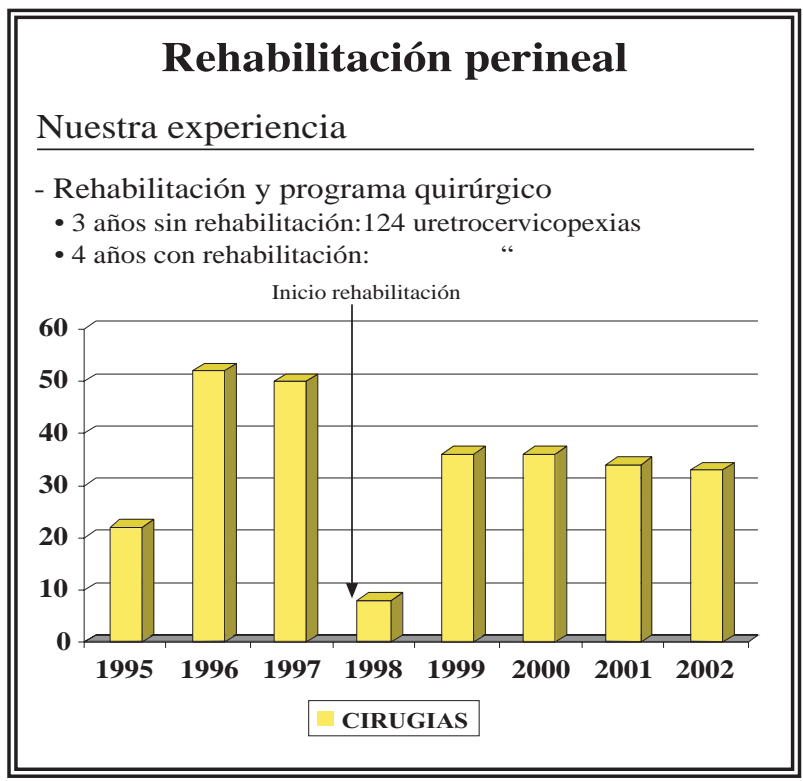

FIGURA 2

contracción y el avance gradual hacia unos conos de mayor peso fortalecen de forma muy eficaz los músculos del suelo pélvico ${ }^{17,18}$. Se empieza a notar una mejoría en el tono de los músculos en unas 2 ó 3 semanas, y un tratamiento completo suele durar entre 2 y 3 meses (Fig. 3).

La experiencia demuestra que sus mejores resultados se obtienen en pacientes que no han rehabilitado su periné con anterioridad, con incontinencias leves y musculatura con un tono basal aceptable. Es aconsejable como medio de recuperación post-parto. Tiene la ventaja de que es un tipo de tratamiento que se ejecuta en domicilio y puede utilizarse también como sistema de valoración perineal previo a otro tipo de tratamiento ${ }^{19,20}$.

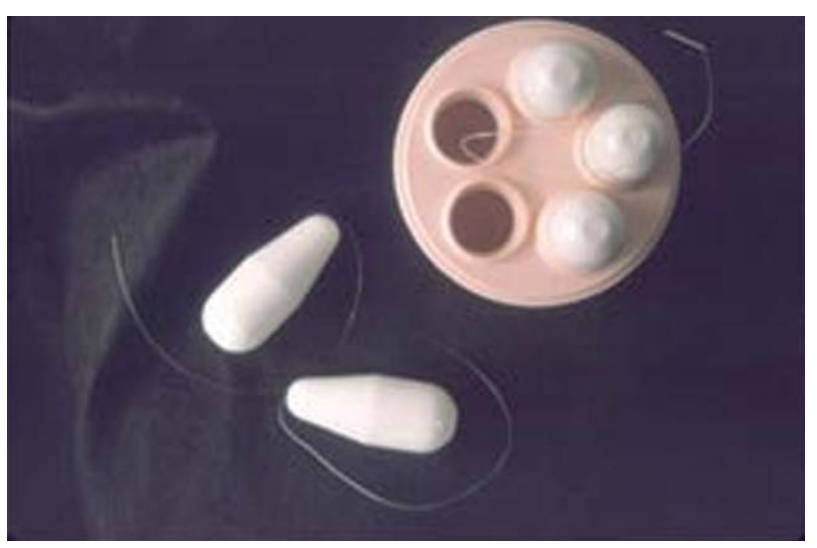

FIGURA 3 
Los resultados son variables según las series, con un rango de éxitos entre el 10 y el 35\%. Se ha publicado una Revisión Cochrane en el 2006 en la que se incluyeron 15 estudios con 1126 mujeres, de las cuales 466 fueron tratadas con conos. Todos los ensayos eran pequeños y, en muchos, fue difícil juzgar la calidad. Las medidas de resultados difirieron entre los estudios, lo que dificultó la combinación de resultados. Los conos fueron mejores que ningún tratamiento activo (RR para el fracaso para curar la incontinencia 0,74; IC del 95\%: 0,59 a 0,93). Esta revisión aporta algunas pruebas de que los conos vaginales pesados son mejores que ningún tratamiento activo en mujeres con incontinencia urinaria por esfuerzo. Sin embargo se trata, hoy en día, de una técnica en desuso.

\section{La rehabilitación pasiva se consigue con la electroestimulación}

Su utilización como forma de tratamiento de la incontinencia urinaria en sus diversas formas viene realizándose desde Caldwell ${ }^{21,22}$ quien implantó los primeros estimuladores en 1963.

Mediante ella se pretende inducir la contracción pasiva del esfinter periuretral y de la musculatura perineal cuando se aplica una corriente eléctrica a través de la pared vaginal o rectal.

Su mecanismo de acción se realiza fundamentalmente a través de la activación refleja, por lo que es imprescindible que el arco reflejo espinal esté íntegro.

Su efecto inmediato es la contracción muscular, consiguiéndose a largo plazo la tonificación e hipertrofia muscular y para algunos el cambio del patrón histológico de la fibra muscular.

En función al tipo de incontinencia a tratar los parámetros de la corriente aplicada variarán de acuerdo al efecto reflejo que deseamos activar.

En IUE, con corrientes superiores a $50 \mathrm{~Hz}$. se consigue activar el reflejo pudendo-pudendo.

En incontinencia de urgencia empleamos frecuencias entre 5 y $10 \mathrm{~Hz}$. que activan el reflejo pudendo inhibitorio del detrusor, produciendo la desaparición o disminución de las contracciones involuntarias.

Las pautas del tratamiento electroestimulador son muy variadas y sus resultados son dis- cordantes debido a la falta de acuerdo a la hora de medir o denominar el éxito o fracaso de la terapia.

Asimismo no existe consenso en su indicaciones ni en el método a aplicar. Ante ello nosotros somos partidarios de realizar rehabilitación activa siempre que exista una respuesta contráctil aunque ésta sea pobre, dejando la electroestimulación únicamente para casos de hipotonía muscular severa, sin sensaciones propioceptivas de contracción. Cuando éstas se recuperan pasamos directamente a la rehabilitación activa mediante biofeedback.

Otra indicación que damos a la electroestimulación es en pacientes incapaces de realizar una contracción al no tener integrado su periné en el esquema corporal. Invitamos a que la paciente contraiga los músculos en el momento en que percibe el estímulo eléctrico, hasta despertar las sensaciones propioceptivas de contracción (Fig. 4).

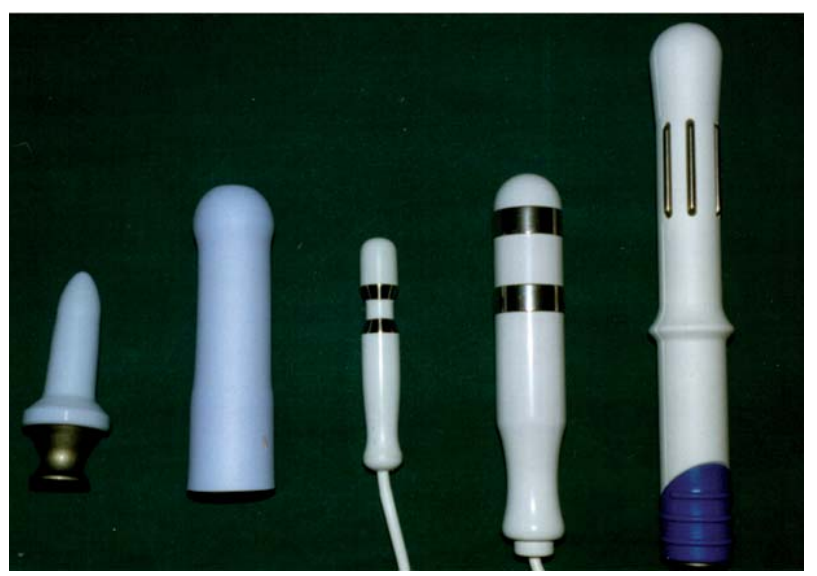

FIGURA 4

\section{BIORRETROALIMENTACION (BIOFEEDBACK)}

La rehabilitación vesical mediante técnicas de BFB la comenzamos a utilizar en el año 1979 cuando nuestros enfermos/as con enuresis e incontinencia de esfuerzo eran sometidos a estudios urodinámicos, con la intención de descartar patología neurológica responsable de su cuadro, pudimos observar que estos pacientes aprendian rápidamente a reconocer con facilidad el trazado electromiográfico y modificarlo voluntariamente consiguiendo que mejorase la sintomatologia miccional cuando acentuaban más o menos su esfuerzo de continencia. 
La continencia de orina se debe a una actividad conjunta y compleja del cuello vesical, esfinter uretral propiamente dicho, la acomodación del detrusor y la musculatura estriada del periné. Esta musculatura y las fibras estriadas del esfinter uretral, reciben su inervación a través de los nervios pudendos, que en principio es de carácter voluntario y por lo tanto susceptible de aprendizaje directo.

Indirectamente la continencia se ve favorecida por la actividad inhibitoria de los centros superiores sobre los centros miccionales sacros, donde se desarrolla e integra la micción.

Este control escapa al aprendizaje directo, pero se puede desarrollar, una vez que los mecanismos voluntarios se establezcan a nivel automático. Aprender es en esencia, crear vías de facilitación para algún tipo de actividad neurológica. Estas vías se consiguen realizando una y otra vez dicha actividad.

La biorretroalimentación es una técnica mediante la cual, procesos fisiológicos normalmente inconscientes se presentan al paciente y al terapeuta como señales de naturaleza visual, táctil o auditiva. La señal se obtiene a partir del parámetro fisiológico y es utilizada posteriormente para enseñar y conseguir así un resultado terapéutico específico. De esta forma, la señal se registra y se muestra de forma cuantitativa y se instruye al paciente para que pueda alterar la señal y a través de esta cambie el proceso fisiológico básico ${ }^{23}$.

En un intento de justificar el mecanismo de acción sobre el que podíamos incidir para incrementar el control del sistema nervioso superior sobre la actividad del detrusor y esfinteriana y cual podría ser el substrato anatomo-fisiológico en el que justificar la realidad del procedimiento. ¿Estábamos obsesionados o existía una posible explicación a nuestras observaciones?

El detrusor y el esfinter interno de la uretra tienen una actividad vegetativa y por lo tanto no están sujetos a la voluntad. Pero también es verdad que, existe una capacidad voluntaria para activar o inhibir el desencadenamiento reflejo de la micción. Además y sobre todo, que la continencia se debe a la actividad conjunta y compleja del cuello vesical, esfinteres, periné y detrusor.
El trabajo de Bradley, Innervation of the male urinary bladder ${ }^{24}$, nos fue de suma utilidad para comprender el mecanismo por el cual podríamos actuar mediante las técnicas de Biofeedback sobre la vejiga y esfinteres, provocando un cambio en la modulación neurológica de los mismos.

Los reflejos miccionales pueden organizarse de acuerdo a los cuatro bucles de integración miccional descritos por Bradley $^{24}$, pudiéndose diferenciar la iniciación del reflejo de vaciamiento, el mantenimiento de la contracción de la vejiga y la terminación del vaciamiento.

Los reflejos que inician el vaciamiento se realizan mediante el Bucle I y II.

Los reflejos que sostienen el vaciamiento, dependen del Bucle II y III y de un feedback positivo aportado por los reflejos uretrovesicales, hay un alargamiento del cuello vesical y un acortamiento de la uretra proximal.

Los reflejos que terminan el vaciamiento, se realizan a través de los Bucles I y IV y de un feedback negativo a los nervios pélvicos, motores del detrusor.

Para nuestros propósitos consideramos como más relevantes los loops IV y III.

En el Loop IV la situación de la musculatura perineal estriada que rodea la uretra, es captada por terminaciones sensitivas del nervio pudendo. Los impulsos aferentes alcanzan las metámeras medulares S-2, S-4 y, ascendiendo por las columnas posteriores van hasta el tálamo y de ahí a la corteza cerebral, en los lóbulos frontales. De aquí desciende una orden motora que por las columnas laterales alcanzará las metámeras sacras S-2, S-4. Desde aquí por el nervio pudendo alcanza la musculatura estriada del periné, cerrando el bucle.

Hay por lo tanto, un posible camino para actuar sobre la continencia de orina, actuando sobre la actividad muscular del periné.

El Loop III es más simple, los impulsos aferentes se originan en los receptores de distensión vesical y siguiendo la vía de los nervios pélvicos alcanzan las metámeras S-2, S-4. Aquí se produce una respuesta refleja, con salida de impulsos motores a través de los nervios pudendos que alcanzan a la musculatura estriada del periné.

En este caso, ante el llenado vesical hay automáticamente, una mayor actividad de los mecanismos de continencia perineales. 
Ejercitando el control voluntario de la musculatura perineal facilitaremos de forma directa el Loop IV e indirectamente el Loop III, que justamente es el que mantiene la continencia cuando la vejiga esta llena.

Pero, además, existen otras vías indirectas, que pueden participar en el aprendizaje:

De una parte tenemos que la inervación vegetativa también alcanza el esfinter externo y por lo tanto va, en alguna forma, a participar en la actividad del mismo. No olvidemos que la sensibilidad y motilidad están intimamente imbricadas en las vías vegetativas.

De otra parte contaremos con los reflejos espinales propio y esteroceptivos, que de hecho establecen una interrelación funcional entre la actividad del suelo de la pelvis y la musculatura lisa de la uretra y vejiga.

Con todo esto intentamos aportar la base interpretativa al mecanismo de acción del biofeedback en la rehabilitación miccional.

De forma específica, el biofeedback es empleado en la educación vesical como tratamiento de la inestabilidad vesical, de la debilidad del suelo pélvico, de la enuresis y de las alteraciones funcionales de la micción tales como la micción no coordinada.

Los objetivos del biofeedback son:

- Captar por algún procedimiento la actividad de los esfinteres y suelo pélvico y hacerla aparente de algún modo al paciente.

- Ensayar como modificar dicha actividad hasta encontrar una respuesta adecuada.

- Repetir los ensayos correctos una y otra vez hasta conseguir automatizarlos.

Antes de iniciar el programa de biofeedback y al igual que en todas las técnicas de reeducación vesical, la paciente recibe una explicación sobre el proceso que motiva sus molestias y sobre el tratamiento al que la vamos a someter, lo que podemos esperar del mismo, la duración del programa y pondremos especial énfasis en solicitar su colaboración activa, secreto del éxito de toda técnica de entrenamiento vesical.

El registro de la actividad de los músculos del suelo pélvico y de los esfinteres se recoge mediante electromiografia, para ello se utilizan electrodos de superficie; antes se colocaban electrodos de aguja, intrarrectales o intrauretrales que actual- mente han quedado obsoletos por resultar más cruentos o más complejos de colocar y mantener en posición ${ }^{25}$. Los electrodos de superficie se colocan a ambos lados del ano, tras secar la zona y localizar el esfinter del ano, se coloca un electrodo a las $3 \mathrm{y}$ otro a las 9 horas y un tercer electrodo de referencia o neutro, actúa como tierra y se coloca en un sitio sin actividad electromiográfica durante la sesión (como por ejemplo un brazo o una pierna).

El registro puede objetivarse de múltiples formas, como señales auditivas o visuales, para ello existen varios equipos en el mercado con multitud de programas que van desde simples líneas que suben o bajan o luces que se encienden progresivamente o sonido que aumenta gradualmente, hasta globos aerostáticos que suben o bajan en una pantalla que simula el cielo o un caballito de mar que nada en el mar, pantallas muy adecuadas cuando vamos a trabajar con niños.

Una vez conectados los electrodos al equipo se invita a la paciente a que contraiga el periné, "contraiga su musculatura como si estuviese orinando y quisiera cortar el chorro" o también, "contraiga sus músculos como si se le fueran a escapar gases y quisiera retenerlos", poco a poco, con paciencia y cariño se le enseña a abandonar la contracción de los rectos abdominales, palpando con una mano en su abdomen y logrando que lo relaje durante la contracción perineal, posteriormente corregiremos la contracción no deseada de la musculatura glútea, diciendo a la mujer que ponga las palmas bajo sus nalgas y note los glúteos relajados mientras contrae el suelo de la pelvis y así sucesivamente con todos los músculos agonistas. Algunos equipos tienen canales accesorios para mostrar a la paciente la contracción perineal y la de la musculatura accesoria que estamos intentando enseñar a que no utilice.

Cuando la paciente aprende una contracción perineal aislada y pura, utilizando exclusivamente los elevadores del ano, comenzamos a enseñar relajación, pues para el tratamiento de la incontinencia de esfuerzo es fundamental la contracción perineal pero en el biofeedback como tratamiento de inestabilidad vesical o micción no coordinada es fundamental la relajación, de esta forma, la relajación del suelo de la pelvis tras contracciones perineales reforzará los hábitos de 
control voluntario de la micción. La paciente puede trabajar de pie, sentada o tumbada según disponibilidad de espacio y mobiliario.

Las sesiones suelen durar unos veinte minutos. Suelen emplearse seis sesiones por paciente, con una periodicidad semanal; aunque esto es variable según el tiempo que tarde una paciente en lograr aislar la contracción de los elevadores del ano y en empezar a obtener resultados. Al final de cada sesión se invita a la paciente a practicar en casa diariamente durante quince minutos por la mañana y por la tarde hasta la nueva sesión. Un diario miccional proporciona refuerzo positivo y ayuda en el cumplimiento de las sesiones. Una vez acabadas las seis semanas de aprendizaje es preciso que la paciente siga en casa algunas semanas más hasta evaluar definitivamente los resultados. Nosotros completamos tres meses de tratamiento antes de tomar otra decisión terapéutica.

Los resultados son variables según las series, llegando hasta el 90\% de mejoría en algunos casos aunque no existe evidencia estadísticamente significativa para recomendar su uso. Se considera que la principal aportación del biofeedback es ayudar al entrenamiento para conseguir una correcta contracción de los músculos del suelo pélvico, sobre todo a mujeres que tienen dificultad inicial; y mantener la motivación en el cumplimiento del programa. En nuestra serie, desde 1997 a 2002, 227 mujeres con incontinencia de urgencia o mixta, sin respuesta a anticolinérgicos ni reeducación vesical, fueron tratadas con biofeedback, tras lo cual el 20\% quedaron secas, y un $40 \%$ adicional con franca mejoría. En otra serie desarrollada también en nuestro Hospital, desde 1995 a 2005 se trataron 680 mujeres con incontinencia de de las que 405 eran de esfuerzo y 105 de urgencia. Para la incontinencia de esfuerzo, el grado de satisfacción de las pacientes, medido según el deseo de operarse, descendió desde $495(82 \%)$ a $170(29,5 \%)$. Para la incontinencia de urgencia descendió desde $89(85 \%)$ a $25(24 \%)$.

\section{ELECTROESTIMULACIÓN / NEUROMODULACIÓN}

\section{Electroestimulación}

El objetivo de la estimulación eléctrica es, según su indicación, mejorar la funcionalidad del suelo pélvico en la incontinencia de esfuerzo e inhibir la hiperactividad detrusoriana en la incontinencia de urgencia. Se utiliza desde hace más de 50 años para tratamiento de la incontinencia; y sin embargo no existe una base biológica clara que explique su mecanismo de acción. Se ha comprobado que es posible conseguir una buena contracción de los músculos del suelo pélvico mediante estimulación del nervio pudendo aplicada con un electrodo vaginal. Por otro lado, en teoría, su fundamento es la capacidad de inhibición del detrusor por medio de la contracción voluntaria de los músculos del suelo pélvico, al estimular estos las fibras simpáticas inhibidoras, que a su vez van a inhibir el centro para-simpático sacro (S2-S4); es el reflejo 12 de Mahoney o reflejo inhibitorio-perineo-bulbar. No obstante hay que señalar que los parámetro óptimos para la estimulación de los músculos del suelo pélvico no están definidos ${ }^{26}$.

Los equipos actuales permiten una amplia variedad de parámetros de estimulación: tipo de corriente, morfologia de la onda, frecuencia, intensidad, localización de los electrodos, etc... Esto contribuye a la dificultad para la comparación de los resultados en las distintas series. No existe consenso internacional para recomendar en un determinado orden la estimulación eléctrica, de modo que cada grupo la utiliza según su propio algoritmo, siempre enmarcado en la modalidad conservadora. En nuestro caso, se indicó electroestimulación en 72 pacientes en el periodo comprendido entre 1997 y 2002. Se aplicó el programa en aquellos que, al iniciar la reeducación, tenían bajo tono perineal o eran incapaces de aprender la mecánica; con un patrón de 6 sesiones de 20 minutos de duración, electro estimulador vaginal y corrientes bifásicas con impulsos de anchura $1000 \mathrm{mcsg}$, frecuencia $5 \mathrm{~Hz}$ e intensidad de menos de 10 mA. De éstas el 66\% fueron rescatadas.

\section{Estimulación eléctrica periférica}

Aunque se trata de una técnica usada desde hace años para el tratamiento de la incontinencia urinaria, la valoración de la literatura científica a este respecto es dificil; en primer lugar, por la ausencia de bases fisiológicas probadas en su método de actuación, y en segundo lugar por la 
falta de consenso en cuanto a los protocolos usados. El objetivo de la estimulación eléctrica es, según su indicación, mejorar la funcionalidad del suelo pélvico en la incontinencia de esfuerzo, e inhibir la hiperactividad detrusoriana en la de urgencia. La estimulación tibial se basa en activar el nervio tibial posterior en el punto descrito por Stoller, que coincide con el SP-6 (siniyinchaio) de la acupuntura, para generar una activación aferente hacia el centro sacro de la micción ${ }^{27}$.

Los equipos actuales permiten una amplia variedad de parámetros de estimulación: tipo de corriente, morfología de la onda, frecuencia, intensidad, localización de los electrodos, etc. En nuestro caso se trató de un equipo SANS de UroSurge, 12 sesiones de 30 minutos, con pulsos de $200 \mathrm{mcsg}, \mathrm{Fc} 20 \mathrm{~Hz}$ e intensidad 1-10 mA.

Se indicó en incontinencia de esfuerzo, en aquellos casos en los que se decidió tratamiento conservador y la paciente presenta incapacidad para la contracción perineal voluntaria; y, por lo tanto, la rehabilitación tradicional es ineficaz. En los casos de incontinencia de urgencia se utilizó cuando no había respuestas a tratamiento farmacológico (anticolinérgicos) ni a medidas tradicionales, incluida la reeducación vesical. La incontinencia mixta la trataremos como la de urgencia, pero con mayor insistencia en la rehabilitación pélvica; sólo si el componente de esfuerzo es predominante o coexiste con un prolapso genital significativo, valoramos antes la corrección quirúrgica del mismo y la uretrocervicosuspensión.

En nuestra serie, desde 1997 a 2003, 80 mujeres con incontinencia de urgencia o mixta sin respuesta a anticolinérgicos, al entrenamiento vesical y al biofeedback, fueron tratadas con electroestimulación, con el equipo que anteriormente he mencionado; siendo clasificados los resultados, respectivamente para percepción del paciente y del médico como excelentes en 11 $(13,7 \%)$ y $9(11,2 \%)$ casos; buenos en $43(53,8 \%)$ y $45(56,3 \%)$; regulares en $14(17,5 \%)$ y $12(15 \%)$ e iguales en $12(15 \%)$ y $14(17,5 \%)$ casos (Fig. 5).

\section{Neuromodulación sacra}

La neuromodulación mediante la estimulación eléctrica de raíces sacras con fines terapéuticos se basa en distintos mecanismos de acción: el
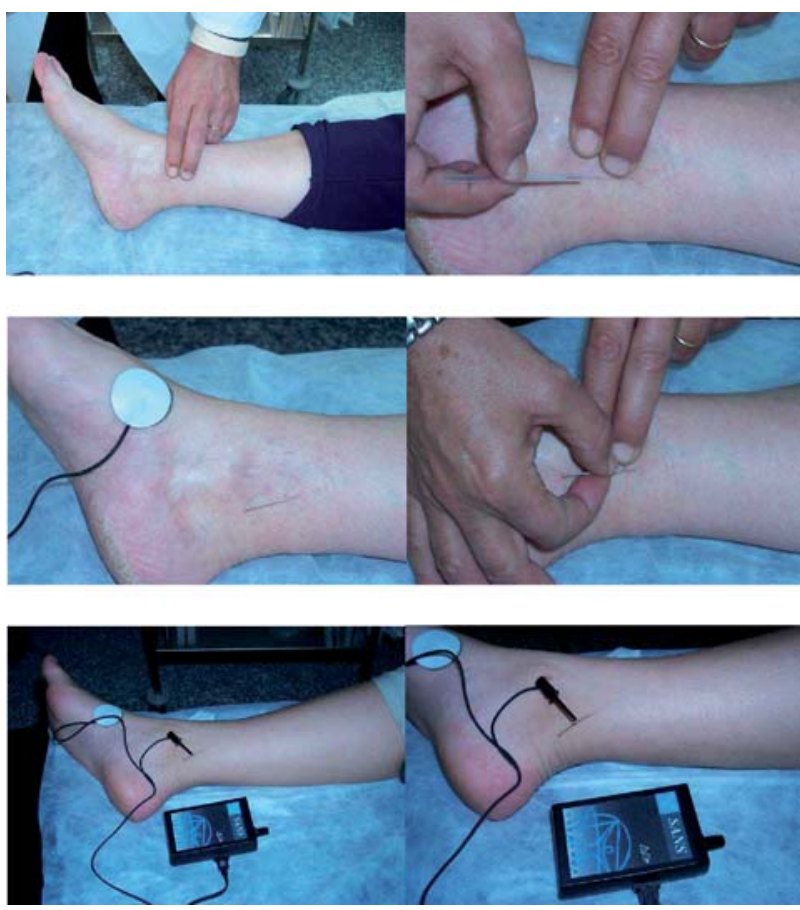

FIGURA 5

central mediante la estimulación de los nervios eferentes (para abolir la inestabilidad vesical mediante la contracción del esfinter uretral externo); y el periférico mediante la creación de un campo eléctrico que estimula las fibras aferentes (mielinizadas tipo A-delta y A-beta somáticas) que transmiten los impulsos sensoriales procedentes de los metámeros correspondientes a las raíces sacras $\mathrm{S} 2-\mathrm{S} 4^{28}$.

Como técnica terapéutica, ha encontrado su lugar en el tratamiento de las disfunciones miccionales crónicas tras el fallo de tratamientos conservadores. Según la FDA las indicaciones son: incontinencia urinaria de urgencia por hiperactividad vesical, asociada a incontinencia fecal o hiperactividad uretral; disfunción miccional del vaciado y sindrome de frecuencia-urgencia asociado o no a dolor pélvico crónico. Y, aunque no aprobadas por la FDA, existen una amplia variedad de otras alteraciones para las que ha sido utilizada: enuresis, disinergia detrusor-esfinter, cistitis intersticial.

Para poder aplicarlas es preciso que el diagnóstico del paciente se ajuste a la indicación, y que tenga capacidad y colaboración para completar los diarios miccionales y los cuestionarios de calidad de vida, exista una respuesta muscular y 
sensorial adecuada en la evaluación de los nervios periféricos. El diagnóstico urodinámico resulta fundamental para determinar el tipo de alteración miccional.

Fases de la neuromodulación sacra:

- Evaluación de raíces sacras. Introducción de un electrodo de estimulación en el foramen sacro S3, que quedará conectado a un generador de estímulos externos 5-7 días, con el objetivo de evaluar la integridad de las fibras somáticas motoras y sensoriales (la respuesta motota es la contracción del suelo pélvico; y la sensitiva es parestesia en periné y genitales externos).

- Fase subcrónica. Determina el efecto terapéutico de la estimulación mediante el diario micccional del paciente, que debe ser de mejoría superior al 50\% respecto de los síntomas iniciales.

- Implante definitivo. Consiste en la colocación de un electrodo con cuatro puntos de estimulación, que clásicamente se coloca a través de una incisión abierta sobre el sacro, bajo anestesia general con relajantes musculares de vida corta (para poder reproducir las respuestas obtenidas en la evaluación de las raíces sacras); y conectado subcutáneamente a un generador de impulsos colocado en el cuadrante supero-externo del glúteo o en la región abdominal anterior. Sin embargo, el alto índice de resultados dudosos de esta técnica ha motivado el desarrollo de un implante percutáneo del electrodo definitivo, que se conecta temporalmente a un generador externo, para evaluar la técnica; y posteriormente se pasa a colocar el generador definitivo de forma subcutánea bajo anestesia local (y por lo tanto con menor tiempo quirúrgico y menos exposición a la fluoroscopia).

El porcentaje de éxitos de esta técnica resulta variable según la indicación para la que se aplique, siendo globalmente del $60 \%$ de mejoría, lo que supone un futuro prometedor de esta técnica para la Neurourología.

\section{REFERENCIAS}

1. Hay-Smith Esc, Bo K, Berghmans LMC, Hendrikg Hjm, de Bier A, van Waalwijk. Pelvis floor muscle trainig for urinary incontinence. Cochrane Incontinence Group. Cochrane Database of Systematic Reviews. Issue 1, 2002.

2. Kegel AH. Progresive resistance exercise in the functionnal restoration of perineal muscle. Am J Obstet Gynecol 1948; 56: $238-248$.
3. Nygaard IE, Kreder KJ, Lepic MM, Fountain KA, Rhomberg AT. Efficacy of pelvic floor muscle exercices in women with stress, urge and mixed urinary incontinence. Am J Obstet Gynecol 1996; 174(1 pt1):120-125.

4. Bourcier A, Juras J. Nonsurgical therapy for stress incontinence. Urol Clin North Am. 1995;22(3):613-627.

5. Medina López RA, Campoy Martínez P, Ramírez Mendoza A, Soltero González A.de la incontinencia urinaria de esfuerzo femenina en una unidad especializada. Actas Urol Esp. 1998; 22:94-102.

6. Kennedy A. Bladder re-education for the promotion of continence. En: Roe B. Clinical Nursing Practice: The promotion and managet al.. Urinary incontinence in adults: acute and chronic management. Clinical practice guideline, № 2. 1996. Rockville: Departament of Health and Human services. Public Health Service, Agency for Health Care policy and Research,

7. Roe B, Williams K, Palmer M. Bladder training for urinary incontinence in adults. En: The Cochrane Library, 2000. 3:

8. Wiseman PA, Malone-Lee J, Rai GS. Terodiline with bladder retraining for detrusor instability in elderly people. BMJ 1991; 302:994-996.

9. Davila Gw, Promozich J. prospective randomized trial of bladder retraining using an electronic voiding device versus self administered bladder drills in women with detrusor instability. Neurourol Urodyn 1998; 17:324-325.

10. Schnelle Jf. Treatment of urinary incontinence in nursing home patients by promted voiding. $\mathrm{J}$ Am Geriatr Soc. 1990;38(3):356-360.

11. Eustice S, Boe B, Paterson J. Promted voiding for the management of urinary incontinence in adults. En: The Cochrane Library, 3:2000. Oxford.

12. Janssen CC, Lagro Janssen Al, Felling AJ. The effects of physiotherapy for female urinary incontinence: individual compared with group treatment. BJU Int. 2001;87(3):201-206.

13. Serra L. Fisioterapia perineal. En: Tratado de reeducación urogineproctológica. Salinas J, Rapáriz M. 263-292. Gráficas Santer SL. Madrid, 1997.

14. BourcierA. Physical therapy female pelvis floor disorders. Curr Opin Obstet Gynecol. 1994;6(4):331-335.

15. Berghmans LC, Frederiks CM, de Bie RA, Weil EH, Smeets LW, van Waalwijk van Doorn ES, Janknegt RA. Efficacy of biofeedback, when included with pelvic floor muscle exercise treatment, for genuine stress incontinence Neurourol Urodyn. 1996;15(1):37-52.

16. Esteban M, Salinas J, Verdejo C, Ramirez Jc, Resel L. Estudio prospectivo de rehabilitación perineoesfinteriana mediante conos vaginales en la incontinencia urinaria de esfuerzo femenina. Toco-Gyn Pract.1996;55:440-450,

17. Bo K. Vaginal weight cones. Theoretical framework. Effect on pelvic floor muscle strength and female stress incontinence. Acta Obstet Gynecol Scand. 1995;74(2):87-92.

18. Peattie AB, Plevnik S, Stanton SL.. Vaginal cones: a conservative method of treating genuine stress incontinence. Br J Obstet Gynaecol 1988;95(10):1049-1053.

19. Pieber D, Zivkovic F, Tamussino K, Ralph G, Lippiitt G, Fauland B. Pelvic floor exercise alone or with vaginal cones for the treatment of mild to moderate stress urinary incontinence in premenopausal women. Int Urogynecol J. 1995;6:14-17.

20. Pieber D, Zivkovic F, Tamussino K. Pelvic floor exercises without or with vaginal cones in premenopausal women with mild to moderate stress incontinente. Gynakol Geburtshilfliche Rundsch. 1994;34(1):32-33. 
21. Fall M, Lindstrom S. Electrical stimulation. Urol Clin North Am.1991;18(2): 393-407.

22. Salinas J, Virseda M. La estimulación eléctrica en el tratamiento de la incontinencia urinaria. En: Urodinámica Clínica $2^{\text {a }}$ edición. Salinas J, Romero J. Jarpyo. Madrid, 1995.

23. Cammu H, Van Nylen M, Amy JJ.. A 10 year follow-up after Kegel pelvic floor muscle exercises for genuine stress incontinence. BJU Int. 2000;85(6):655-658.

24. Bradley WE.: Innervation of the male urinary bladder. Urol Clin North Am. 1978;5(2):279-293.

25. Espuña, M, Salinas, J. Tratado de Uroginecología. Ars Medica 2004; 33:449-457,. Barcelona.

26. Hunskaar S, Emery S, Jeyaseelan S. Electrical stimulation for urinary incontinence in woman. Cochrane Incontinence Group. Cochrane Database of Systematic Reviews. Issue 1, 2002.
27. Berghmans B, van Waalwijk van Doorn E, Nieman F, de Bie R, van den Brandt P, Van Kerrebroeck P. Efficacy of physical therapeutic modalities in women with proven bladder overactivity. Eur Urol. 2002,; 41(6):581-587.

28. Aboseif S, Tamaddon K, Chalfin S, Freedman S, Kaptein J. Sacral neuromodulation as an effective treatment for refractory pelvic floor dysfunction. Urology 2002;60(1):52-56.

Correspondencia autor: Dr. J.M ${ }^{\mathrm{a}}$ Pena Outeiriño Unidad de Neurourología y Uroginecología.

Hospital Universitario Virgen del Rocío.

Avda. Manuel Siurot, s/n - 41013 Sevilla Tel.: 955012000

E-mail autor: penaespina@yahoo.es

Información artículo: Revisión - Urología funcional

Trabajo recibido: abril 2007

Trabajo aceptado: mayo 2007 